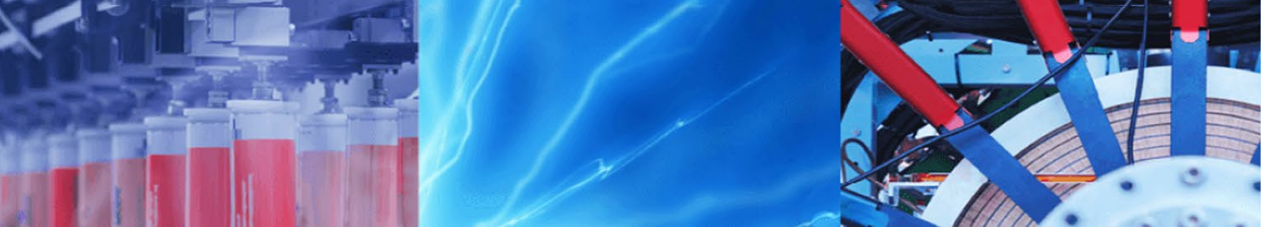

Research Article

\title{
Stability analysis of the homogeneous nonlinear dynamical systems using Lyapunov function generation based on the basic functions
}

\author{
Mehdi Ansari $^{1}\left[\right.$ Vahid Meigoli $^{1} \cdot$ Abolhassan Razminia $^{2}$
}

Received: 31 October 2019 / Accepted: 8 January 2020 / Published online: 16 January 2020

(c) Springer Nature Switzerland AG 2020

\begin{abstract}
In this paper, based on Lyapunov functions candidates, a new approach in the stability analysis of homogeneous nonlinear systems is proposed in which instead of concentrating on the positive definiteness of the Lyapunov candidate functions, we stress on the negative definiteness of its derivative. Having ensured of negative definiteness of the derivative function, based on sign assignment of the primitive function, the stability of the equilibrium is analyzed wherein the necessary and sufficient conditions are declared simultaneously. Selecting the trend of the Lyapunov candidate function is primarily performed in the form of a linear combination of some simple functions whose unknown coefficients in the candidate function structure are computed based on negative definiteness of the derivative function. Afterward, using these determined coefficients in the Lyapunov function, the sign of the primitive function in the state space is argued. Therefore, the triple sign attitudes of the candidate function can be used to deduce the stability/instability of the equilibrium point. Moreover, in the process of the negative definiteness of the derivative function, the coefficients are obtained using two independent methods. Numerical simulations support the proposed theoretical results and show their effectiveness.
\end{abstract}

Keywords Stability analysis · Lyapunov function · Homogeneous nonlinear dynamical systems · Least squares method

\section{Introduction}

Today, the importance of the issue of stability in control systems, both linear and nonlinear, is clear to everyone, so many researchers' efforts in the field of control systems have devised appropriate methods for analyzing the stability of control systems [1]. One of the applications of the stability analysis is in cyber-physical systems (CPS) [2], so the stability analysis for these systems is more critical because, as mentioned in [3-5], these systems exposure with many attacks. In other industrial systems also, stability is a vital factor such as breakwater [6], structural reliability analysis [7], inverter-based nonlinear power systems [8], also some new advances in this field as [9], and so on. As we know, the main idea behind the stability analysis of dynamical systems is the Lyapunov methods. Given that the first method of Lyapunov, utmost opines on the local stability of an equilibrium point, in practice, for extremely nonlinear systems, such as chaotic systems, is not so practical.

On the other hand, the second or direct Lyapunov method, although presenting a sufficient condition for the stability of the equilibrium point of a nonlinear system but finding a candidate function is not systematic or cumulative to meet the conditions of Lyapunov and depending on the type of problem, some functions are offered using trial and error.

\footnotetext{
$\triangle$ Mehdi Ansari, m.ansari@mehr.pgu.ac.ir | 'Department of Electrical Engineering, School of Engineering, Persian Gulf University, Bushehr, Iran. ${ }^{2}$ Dynamical Systems \& Control (DSC) Research Laboratory, Department of Electrical Engineering, School of Engineering, Persian Gulf University, P.O. Box 75169, Bushehr, Iran.
} 
So far, many attempts have been made to provide a method for selecting the Lyapunov function, each of which has disadvantages and advantages. For example, we can mention the following: In [10], the method of forming a Lyapunov function with the aid of linear programming for autonomous systems is provided. The mentioned method also provides a lower bound for the absorption region. Establishing a Lyapunov function in the square form for polynomial systems of positive dimensions has taken in [11]. The square Lyapunov function defined in this method is such that some coefficients are unknown, which was calculated using the Homotopy continuation algorithm. A method to determine the Lyapunov function for the desired switching dynamical systems is given in [12]. In [11], by using the theory of normal forms, a method for determining the Lyapunov function for nonlinear systems is presented in the form of normal coordinates.

One of the new ideas in the field of the choice of the Lyapunov function for analyzing the stability of the point of equilibrium of nonlinear systems is the method reported in $[13,14]$. This way, called the sum of squares method, or SOS, by forming the Lyapunov function as a sum of pairwise powers polynomial expressions, constructs a certain positive function. Ultimately, by solving a convex optimization problem calculates the unknown coefficients. Recently, the mentioned method has been numerically developed for both types of continuous and discrete-time systems in [15].

Also, in [16], a general structure for the stability analysis of nonlinear systems is presented based on the Sum Of Squared method, which takes place base on the decomposition of the vector into another system. That system is explained in the form of a polynomial vector field with feeds with no memory sentences. It should be noted that all these methods of calculating the Lyapunov function are based on a certain positive function in the sum form squares that simultaneously, the derivative of this function gives a symmetric representation of a sum of squares.

In [17], the backstepping approach is considered for a class of block strict-feedback nonlinear systems. Based on the assumption that nonlinear systems are polynomials, for each backstepping step, the Lyapunov function can be constructed in a polynomial form by Sum Of Square (SOS) technique. A specific linear combination of subsystems' energies is proposed as Lyapunov function for multi-degree-of-freedom nonlinear stochastic dynamical systems, and the corresponding sufficient condition for the asymptotic Lyapunov stability with probability one is then determined [18]. In [19], they presented a methodology for the algorithmic construction of Lyapunov functions for the transient stability analysis of classical power system models. The proposed methodology used recent advances in the theory of positive polynomials, semidefinite programming, and sum of squares decomposition. In [20] they proposed an approach constructed a continuous piecewise affine function given a suitable partition of the state space, called a triangulation, and values at the vertices of the triangulation. The vertex values are obtained from a Lyapunov function in a classical converse Lyapunov theorem.

Recently in [21], we introduced a new approach to constructing the Lyapunov function, which was fundamentally different from existing methods. In fact, we began with a primary focus on defining the derivative of a function, and then, in the domain space, we define the sign of the candidate function itself. In this way, the result was expressed as a necessary and sufficient condition for the stability of the equilibrium point. The main distinction of this method with the other methods is starting with $\dot{V}$ and then reaching $V$, while other methods start from a $V$ as the candidate of Lyapunov's function and finally, by determining $\dot{V}$ opines on the stability of the equilibrium point. In addition, another innovation in that paper is to provide Lyapunov functions based on the linear combination of simple functions whose coefficients were computed analytically. As a difference with the sum of squares method, calculation of $V$ and $\dot{V}$ in sum of square method was done at the same time to become sure about the proper symbols of each of these two functions.

In this paper, we extend the method of [21] and calculate the coefficients of Lyapunov function numerically, so many more problems could be solved. The coefficients are determined by using the derivation of the above function. It is worth noting that our main study in this paper deals with standard homogeneous nonlinear systems that are used today in many of the science and engineering branches [22-24]. Our motivation for the use of these kinds of systems is the formation of candidate Lyapunov function in the form of a linear combination of sentences of the same degree. This candidate function structure, as we will see, will get a systematic approach to stability analysis that will be considered one of the other innovations in this article.

We have to say the method of calculation of unknown coefficients in the SOS approach is fundamentally based on convex optimization, while in this paper, we use two simple algorithms based on Least Squares to calculate the unknown coefficients.

The paper structure is as follows: After this brief introduction, in Sect. 2, the theoretical prerequisites provided for the homogeneous nonlinear systems are reviewed. The main theoretical outcomes of the paper are presented in Sect. 3. In order to demonstrate the ability of the developed theory, some examples are given in Sect. 4, whose numerical simulations confirm the validity of the 
theoretical results. Finally, the conclusion and the overall results close the paper in Sect. 5.

\section{Homogeneous systems}

In this paper, a special class of nonlinear systems, called standard homogeneous nonlinear systems, is studied. Homogeneous polynomial differential equations appear in all fields of science and engineering due to their unique features.

In fact, it can be said in a general language that many terminology and analytical points in the field of linear systems can be expanded to nonlinear homogeneous systems. One of these unique features is the equivalence of many local attributes around the equilibrium point and globality. For example, if the equilibrium point of a homogeneous nonlinear system is locally stable, in this case, it is also global stable. This feature and other features have led to the widespread use of these systems in the modeling and description of physical systems.

Definition 1 ([25]) A polynomial, whose all terms have the same degree, is called homogeneous polynomial; in other words, a $V(X): \mathbb{R}^{n} \rightarrow \mathbb{R}$ function is homogeneous of $p$ degree whenever we hold for all $\lambda \in \mathbb{R}$ :

$V(\lambda \mathbf{x})=\lambda^{p} V(\mathbf{x})$

In this case, we write $V \in \mathrm{H}_{\mathrm{p}}$.

Definition 2 ([25]) A dynamical system $\dot{\mathbf{x}}=\mathbf{f}(\mathbf{x})$ is homogeneous of $k$ degree if for $\mathbf{f}(\mathbf{x})=\left[\mathrm{f}_{1}(\mathbf{x}) \mathrm{f}_{2}(\mathbf{x}) \ldots \mathrm{f}_{\mathrm{n}}(\mathbf{x})\right]^{\top}$ vector field and $\lambda \in \mathbb{R}$ we have:

$\mathbf{f}(\lambda \mathbf{x})=\lambda^{k} \mathbf{f}(\mathbf{x})$

In this case, we write $f \in S_{k}$.

It can be seen from Eq. (1) if the homogeneous polynomial $V$ has the same sign on an arc of the unit circle $\left\{\mathbf{x} \in \mathbb{R}^{\mathrm{n}}: \mathbf{x}=1\right\}$, in this case, that function will be the same on the radial part of that arc. This feature is shown in Fig. 1. As an example, the yellow sector is, in fact, the expansion of a branch of a corresponding arc, in which the polynomial does not change its sign on it. Thus, the problem of determining the sign of a homogeneous polynomial decreases to its sign on a single circle.

Definition 3 For a vector such as $\mathrm{x} \in \mathbb{R}^{\mathrm{n}}$ and a natural number such as $p \in \mathbb{N}$, $p$-form representation is defined as follows:

$\mathbf{x}^{[p]}=\left(x_{1}^{p}, x_{1}^{p-1} x_{2}, x_{1}^{p-1} x_{3}, \ldots, x_{1}^{p_{1}} x_{2}^{p_{2}} \ldots x_{n}^{p_{n}}, \ldots, x_{n}^{p}\right)$

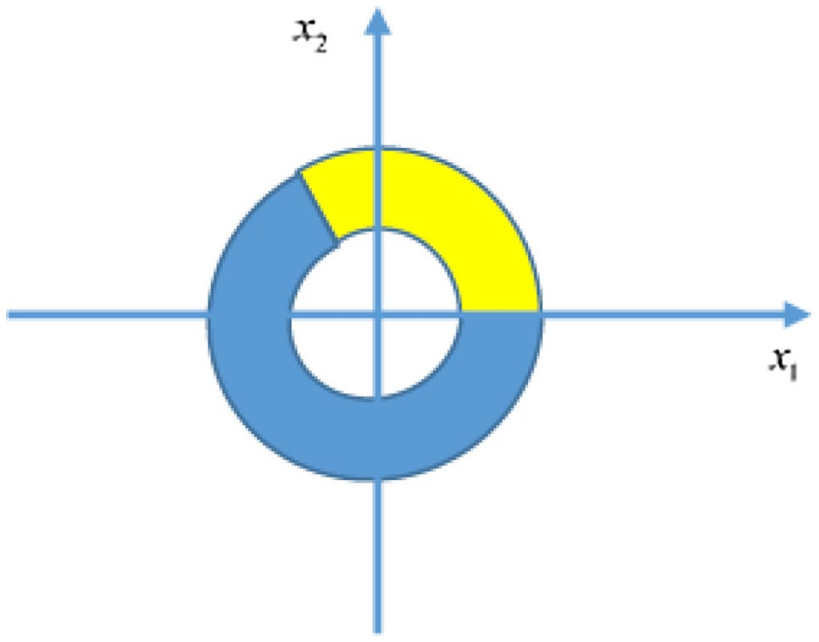

Fig. 1 Showing the no-sign-change of a homogeneous polynomial in a sector expansion

This $p$-form representation contains sentences as $x_{1}^{P_{1}} x_{2}^{P_{2}}, \ldots, x_{n}^{P_{n}}$ which

$p_{1}+p_{2}+\cdots+p_{n}=p, \quad p_{i} \in \mathbb{N}_{0}$

where $\mathbb{N}_{0}=\mathbb{N} \bigcup\{0\}$.

To form the $\mathbf{x}^{[P]}$ representation in a standard manner, we can use lexicography. For example, for $p=5$ and $n=3$, its lexicographic arrangement is as follows:

$$
\begin{aligned}
x^{[5]}= & \left(x_{1}^{5}, x_{1}^{4} x_{2}, x_{1}^{4} x_{3}, x_{1}^{3} x_{2}^{2}, x_{1}^{3} x_{2} x_{3}, x_{1}^{3} x_{3}^{2},\right. \\
& x_{1}^{2} x_{2}^{3}, x_{1}^{2} x_{2}^{2} x_{3}, x_{1}^{2} x_{2} x_{3}^{2}, x_{1}^{2} x_{3}^{3}, x_{1} x_{2}^{4}, x_{1} x_{2}^{3} x_{3}, \\
& \left.x_{1} x_{2}^{2} x_{3}^{2}, x_{1} x_{2} x_{3}^{3}, x_{1} x_{3}^{4}, x_{2}^{5}, x_{2}^{4} x_{3}, x_{2}^{3} x_{3}^{2}, x_{2}^{2} x_{3}^{3}, x_{2} x_{3}^{4}, x_{3}^{5}\right)
\end{aligned}
$$

It can be easily shown for integers $p, n$, there exists $m=\left(\begin{array}{c}n+p-1 \\ p\end{array}\right)$ functions of a single sentence of $p$ degree in a $p$-form representation. Below are some useful features of the homogeneous dynamic systems that are used in the next section. In all of the following, it is assumed that the system equilibrium point is at the origin.

Lemma 1 [26] Assume that the dynamic system $\dot{\mathbf{x}}=\mathbf{f}(\mathbf{x})$ is homogeneous. If the vector field $\mathbf{f}$ is continuous and the equilibrium point of the system is stable, then there is a homogeneous Lyapunov function to prove the stability of this system.

This lemma actually limits the search scope to the Lyapunov function for homogeneous functions, which can be valuable from the computational point of view. An exhaustive version of this proposition is given in the following lemma. 
Lemma 2 [27] For the homogeneous dynamical system $\dot{\mathbf{x}}=\mathbf{f}(\mathbf{x})$ which $\mathbf{f} \in \mathrm{S}_{\mathrm{k}}$ if $\mathrm{V} \in \mathrm{H}_{\mathrm{p}}$ is chosen, then the derivative $V(\mathbf{x})$ along the answers of the following system is a homogeneous function of degree $p+k-1$; that is $\dot{V} \in H_{p+k-1}$.

\section{Theoretical results}

The most famous theorem in analyzing and designing nonlinear systems is the Lyapunov theorem (Lyapunov's direct method), which Provides sufficient conditions for stability of the equilibrium point. Moreover, in [28], the socalled instability theory stated that it shows the unstable equilibrium point for unstable systems. In this section, the concept of equilibrium stability is commented upon by starting with a negative definite term for the derivation of the Lyapunov candidate function and then determining the sign of the function itself.

Theorem 1 The dynamical system $\dot{\mathbf{x}}=\mathbf{f}(\mathbf{x})$ is assumed. If there is a continuous partial function $V(\mathbf{x})$ with $V(0)=0$ and $\dot{V}(\mathbf{x})$ along the system responses is negative definite, in this case if $V(X)$ is topically positive then the equilibrium point of the system is asymptotically stable; otherwise, the point of equilibrium is unstable.

Proof For the given dynamical system, we first show that the function $V(\mathbf{x})$ is never Positive semi definite or negative semi definite. With contrary assumption, if $V(\mathbf{x})$ is a positive semi definite, then at a point out of origin $\mathbf{x}_{0}$, we have $V\left(\mathbf{x}_{0}\right)=0$,.But because of the assumption of the theorem, out of origin, $\dot{V}(\mathbf{x})$ is always negative, and $V(\mathbf{x})$ chances sign by passing that point $x_{0}$ and this contradicts with positive semi definite of the $V(\mathbf{x})$ function. So the hypothesis is invalidated. In the same way, it can be shown that $V(\mathbf{x})$ is never negative semi definite.

Now we can say that $V(\mathbf{x})$ has three states: totally positive definite, totally negative definite, and indeterminate. Each of these three modes is as follows separately reviewed:

1. Situation $V(\mathbf{x})>0$ : This condition is exactly the expression of the Lyapunov theorem, which states that for a positive function $V(\mathbf{x})$, whose derivative is always negative definite, the asymptotically stability of the equilibrium point is deductible.

2. Situation $V(\mathbf{x})<0$ : In this situation, by setting $\Lambda=-V$, clearly the hypotheses of the instability theory are obtained. In other words, for the dynamical system studied, we have: $\Lambda>0$ and $\dot{\Lambda}>0$. Thus, according to [28], the instability of the equilibrium point results.
3. $V(\mathbf{x})$ indeterminate situation: The change of $\operatorname{sign} V(\mathbf{x})$ around the equilibrium point means that there is a region around this point, which on that area the sign of $V(\mathbf{x})$ is negative. For this situation, this small area that is adjacent to the equilibrium point, the $\operatorname{sign} V(\mathbf{x})$ and $\dot{V}(\mathbf{x})$ are both negative, which, according to condition 2 , we conclude that the equilibrium point is unstable.

This completes the proof.

In the following, by limiting the discussion to homogeneous systems, two methods are proposed for the formation of the Lyapunov function, and hence we conclude the globally stability of the equilibrium point by using that way. For this purpose, consider the homogeneous nonlinear dynamical system $\dot{\mathbf{x}}=\mathbf{f}(\mathbf{x})$ that $f \in \mathrm{S}_{\mathrm{k}}$. We consider the basic functions $V_{i}(\mathbf{x})$ in the $i$ th entry of $p$-representation corresponding to state vector $\mathbf{x} \in \mathbb{R}^{\mathrm{n}}$ as follows:

$v_{i}(\mathbf{x})=\mathbf{x}_{i}^{[p]}$

We now construct the function of the Lyapunov candidate in the form of the linear combination of these basic functions:

$$
\begin{array}{r}
V(\mathbf{x})=\sum_{i=1}^{m} a_{i} v_{i}(\mathbf{x}) \\
=\mathbf{a}^{T}\left(\mathbf{x}^{[p]}\right)^{T}
\end{array}
$$

where $m=\left(\begin{array}{c}p+k-1 \\ p\end{array}\right)$ is the representation of the number of terms in $p$-form, and $a_{i}$ are also disturbing coefficients that must be calculated as it goes on in the following. It is noteworthy that the $p$-form exhibitions are considered to be linearly independent. In this case, the linear composition coefficients in Eq. (7) are unique. On the other hand, it is easy to see $V(0)=0$. It is clear that according to the symbol introduced in Eq. (7), the vector of coefficients is as follows

$\mathbf{a}=\left[\begin{array}{llll}a_{1} & a_{1} & \cdots & a_{m}\end{array}\right]^{T}$

Now, by derivation of the proposed Lyapunov Eq. (7) for the homogeneous system $\dot{\mathbf{x}}=\mathbf{f}(\mathbf{x})$ we have:

$\dot{V}(\mathbf{x})=\sum_{i=1}^{m} a_{i} \dot{v}_{i}(\mathbf{x}) \equiv \mathbf{a}^{T} H^{T}\left(\mathbf{x}^{[p+k-1]}\right)^{T}<0$

where $H$ is an intermediate matrix that can be calculated depending on the problem. It should be noted that according to Lemma 2 , we can say that $\dot{V}(X)$ is homogeneous of degree $p+k-1$, and also the linear combination of the base functions $V_{i}^{\prime}(X)$ according to Eq. (9) is homogeneous of the $p+k-1$ degree. The method of calculation of 
the middle matrix is given in the next section. Therefore, the problem becomes the computation of $a$ vector such that Eq. (9) holds.

To do this, we set $\dot{V}(\mathbf{x})$ equal to a definite negative function and calculate the unknown coefficients. Before proceeding to demonstrate general methods, the subject is explained by an example. In order to this issue, first consider the following dynamic system with the equilibrium point of the origin:

$\left\{\begin{array}{l}\dot{x}_{1}=-x_{1}^{3}-2 x_{2}^{3} \\ \dot{x}_{2}=3 x_{1}^{3}-3 x_{2}^{3}\end{array}\right.$

In the subsequent lines the status of the stability of the equilibrium point is analyzed. It is clear that the mentioned system is homogeneous. So we can choose the Lyapunov function, as a homogeneous form of degree 4:

$V(\mathbf{x})=a_{1} x_{1}^{4}+a_{2} x_{1}^{3} x_{2}+a_{3} x_{1}^{2} x_{2}^{2}+a_{4} x_{1} x_{2}^{3}+a_{5} x_{2}^{4}$

and calculate its derivation along the paths of the system:

$$
\begin{aligned}
\dot{V}(\mathbf{x})= & \left(-4 a_{1}+3 a_{2}\right) x_{1}^{6}+\left(-3 a_{2}+6 a_{3}\right) x_{1}^{5} x_{2} \\
& +\left(-2 a_{3}+9 a_{2}\right) x_{1}^{4} x_{2}^{2}+\left(-8 a_{1}-3 a_{2}-a_{4}\right. \\
& \left.+12 a_{5}\right) x_{1}^{3} x_{2}^{3}+\left(-6 a_{2}-6 a_{3}\right) x_{1}^{2} x_{2}^{4} \\
& +\left(-4 a_{3}-9 a_{4}\right) x_{1} x_{2}^{5}+\left(-2 a_{4}-12 a_{5}\right) x_{2}^{6}
\end{aligned}
$$

By putting the above phrase equivalent with a negative phrase like $-12 x_{1}^{6}-24 x_{2}^{6}$, simply the following unique answers are obtained for the coefficients:

$a_{1}=3, a_{2}=a_{3}=a_{4}=0, a_{5}=2$

Thus, the following Lyapunov function is obtained:

$V(x)=3 x_{1}^{4}+2 x_{2}^{4}$

which clearly indicates the asymptotic stability of the equilibrium point.

In this example, as we have seen, the number of necessary equations was also sufficient from which the unique factors of the unknown coefficients were obtained. And the Lyapunov function was obtained. But in general, one cannot be sure that the equations derived from the unification of derivative function with a desired negative definite phrase give a unique answer. In the following, we explain the above example in a more general way; through that way, a method for calculating the Lyapunov function is obtained. The method presented below is simply extendible for more general systems.

\subsection{Algebraic method}

Consider the following nonlinear homogeneous system of degree 3 $\left\{\begin{array}{l}\dot{x}_{1}=b_{11} x_{1}^{3}+b_{12} x_{2}^{3} \\ \dot{x}_{2}=b_{21} x_{1}^{3}+b_{22} x_{2}^{3}\end{array}\right.$

Initially, by choosing a homogeneous Lyapunov candidate function of degree 4, as follows:

$$
\begin{aligned}
V(\mathbf{x}) & =a_{1} x_{1}^{4}+a_{2} x_{1}^{3} x_{2}+a_{3} x_{1}^{2} x_{2}^{2}+a_{4} x_{1} x_{2}^{3}+a_{5} x_{2}^{4} \\
& =\mathbf{a}^{T}\left(\mathbf{x}^{[4]}\right)^{T}
\end{aligned}
$$

By taking time derivative of this function one can get:

$$
\begin{aligned}
\dot{V}(\mathbf{x})= & 4 \dot{x}_{1} x_{1}^{3} a_{1}+\left(3 \dot{x}_{1} x_{1}^{2} x_{2}+x_{1}^{3} \dot{x}_{2}\right) a_{2} \\
& +\left(2 \dot{x}_{1} x_{1} x_{2}^{2}+2 x_{1}^{2} \dot{x}_{2} x_{2}\right) a_{3} \\
& +\left(\dot{x}_{1} x_{2}^{3}+3 x_{1} \dot{x}_{2} x_{2}^{2}\right) a_{4}+4 \dot{x}_{2} x_{2}^{3} a_{5}
\end{aligned}
$$

Now, inserting the dynamics of Eq. (15) in $V$, the above statement reduces to:

$$
\begin{aligned}
\dot{V}(\mathbf{x})= & \left(4 b_{11} a_{1}+b_{21} a_{2}\right) x_{1}^{6}+\left(3 b_{11} a_{2}+2 b_{21} a_{3}\right) x_{1}^{5} x_{2} \\
& +\left(2 b_{11} a_{3}+3 b_{21} a_{2}\right) x_{1}^{4} x_{2}^{2}+\left(4 b_{12} a_{1}+b_{22} a_{2}+b_{11} a_{4}\right. \\
& \left.+4 b_{21} a_{5}\right) x_{1}^{3} x_{2}^{3}+\left(3 b_{12} a_{2}+2 b_{22} a_{3}\right) x_{1}^{2} x_{2}^{4} \\
& +\left(2 b_{12} a_{3}+3 b_{22} a_{4}\right) x_{1} x_{2}^{5}+\left(b_{12} a_{4}+4 b_{22} a_{5}\right) x_{2}^{6}
\end{aligned}
$$

which is retrieved in the following matrix form:

$\left[\begin{array}{l}a_{1} \\ a_{2} \\ a_{3} \\ a_{4} \\ a_{5}\end{array}\right]^{T}\left[\begin{array}{ccccccc}4 b_{11} & 0 & 0 & 4 b_{12} & 0 & 0 & 0 \\ b_{21} & 3 b_{11} & 0 & b_{22} & 3 b_{12} & 0 & 0 \\ 0 & 2 b_{21} & 2 b_{11} & 0 & 2 b_{22} & 2 b_{12} & 0 \\ 0 & 0 & 3 b_{21} & b_{11} & 0 & 2 b_{22} & b_{12} \\ 0 & 0 & 0 & 4 b_{21} & 0 & 0 & 4 b_{22}\end{array}\right]\left[\begin{array}{c}x_{1}^{6} \\ x_{1}^{5} x_{2} \\ x_{1}^{4} x_{2}^{2} \\ x_{1}^{3} x_{2}^{3} \\ x_{1}^{2} x_{2}^{4} \\ x_{1} x_{2}^{5} \\ x_{2}^{6}\end{array}\right]=\mathbf{a}^{T} H^{T}\left(\mathbf{x}^{[6]}\right)^{T}$

where

$H=\left[\begin{array}{ccccc}4 b_{11} & b_{21} & 0 & 0 & 0 \\ 0 & 3 b_{11} & 2 b_{21} & 0 & 0 \\ 0 & 0 & 2 b_{11} & 3 b_{21} & 0 \\ 4 b_{12} & b_{22} & 0 & b_{11} & 4 b_{21} \\ 0 & 3 b_{12} & 2 b_{22} & 0 & 0 \\ 0 & 0 & 2 b_{12} & 2 b_{22} & 0 \\ 0 & 0 & 0 & b_{12} & 4 b_{22}\end{array}\right]$

Now we equalize the Eq. (19) with a certain negative function, such as $Z^{T}\left(\mathbf{x}^{[p+k-1]}\right)^{T}$ :

$\dot{V}(\mathbf{x}) \equiv Z^{T}\left(\mathbf{x}^{[p+k-1]}\right)^{T}$

For example, in the two-variable mode $n=2$, the choice for $Z$ can be as follows:

$Z=\left[\begin{array}{lllllll}-1 & 0 & -1 & 0 & \cdots & 0 & -1\end{array}\right]^{T}$ 
Therefore, by comparing Eq. (19) with Eq. (21), the unknown coefficients $a$ are obtained by solving the following equation.

$H \mathbf{a}=Z$

According to the coefficients of the basic functions, since in the resulting Eq. (23) the number of unknowns $\left(a_{i}\right)$ (the vector length of $x^{[p]}$ ) is less than the number of equations (that is, the vector length of $x^{[p+k-1]}$ ) and the coefficients are not exactly determined, one way to determine the optimal of these abnormalities is to use The least squares method [29], which proposes the following response from Eq. (23)

$\mathbf{a}=\left(H^{T} H\right)^{-1} H^{T} Z$

After calculating the $a^{\prime} s$ coefficients a sign for $\dot{V}(\mathbf{x})$ should be checked out. In case of failure to get the necessary signs two ways are suggested:

1. Reselection the $Z$ vector and then check the necessary sign for the $\dot{V}(\mathbf{x})$ and assign the $V(\mathbf{x})$ sign.

2. Increase the degree of Lyapunov's function and then repeat the steps of the above algorithm.

\subsection{Geometric method}

In the following, another method is presented for calculating the unknown coefficients of the Lyapunov candidate function, based on Theorem 1. For this purpose, consider $V(\mathbf{x})$ to the form of the Eq. (7), we set the value of the function $\dot{V}(\mathbf{x})$ on the unit sphere to a given value. According to the homogeneity of this function, we consider the value of $\dot{V}(\mathbf{x})=-1$. Now, by choosing the number of $N$ points on the surface of the unit sphere, we form the following equations:

$\sum_{i=1}^{m} a_{i} \dot{v}_{i}\left(x^{j}\right)=-1, \quad j=1,2,3, \ldots, N$

where $\mathbf{x}^{j}$ is the j-point on a unit sphere, and we have $N$ number of equations and $m$ unknown parameters, which always must $N \geq m$ that the set of Equations be solvable. Note that these $N$ points are selected in the form of an equal align on the sphere that by increasing their number, points location becomes closer together, and then the precision of the answer gets better. On the other hand, increasing of $N$ will result in heavier computing. That it will slow down the program. The set of Eq. (25) can be retrieved in the following matrix:
$G \mathbf{a}=\left[\begin{array}{cccc}\dot{v}_{1}\left(\mathbf{x}^{1}\right) & \dot{v}_{2}\left(\mathbf{x}^{1}\right) & \cdots & \dot{v}_{m}\left(\mathbf{x}^{1}\right) \\ \dot{v}_{1}\left(\mathbf{x}^{2}\right) & \dot{v}_{2}\left(\mathbf{x}^{2}\right) & \cdots & \dot{v}_{m}\left(\mathbf{x}^{2}\right) \\ \vdots & \vdots & \ddots & \vdots \\ \dot{v}_{1}\left(\mathbf{x}^{N}\right) & \dot{v}_{2}\left(\mathbf{x}^{N}\right) & \cdots & \dot{v}_{m}\left(\mathbf{x}^{N}\right)\end{array}\right]\left[\begin{array}{c}a_{1} \\ a_{2} \\ \vdots \\ a_{m}\end{array}\right]=\left[\begin{array}{c}-1 \\ -1 \\ \vdots \\ -1\end{array}\right]$

where $G$ matrix is a $N \times m$ matrix. Given that in this device, the number of equations is greater than the number of unknowns, one method of calculating the missing vector is using the Least Squares method. Simply the answer is as following:

$\mathbf{a}=\left(G^{T} G\right)^{-1} G^{T}\left(\begin{array}{c}-1 \\ -1 \\ \vdots \\ -1\end{array}\right)$

After calculating a, vector of the coefficients, by inserting them in the $\dot{V}(\mathbf{x})$ expression again, we must check the $\operatorname{sign} \dot{V}(\mathbf{x})$ and $V(\mathbf{x})$. After assurance of the negative making of $\dot{V}(\mathbf{x})$, by using the calculated coefficients $a$ of Eq. (27) and their replacement in Eq. (7), function $V(\mathbf{x})$ forms that by determining its sign and using Theorem 1 , we can analyze the stability of the equilibrium point of the studied system.

\section{Examples and simulations}

In this section, examples are presented to illustrate the theoretical results obtained in the preceding sections. In fact, we will investigate all three modes that are expressed in theory 1 with three different examples using the proposed solution methods.

Example 1 Consider the system introduced in Eq. (10) once again. By choosing the Lyapunov function as in Eq. (16) which is homogeneous of degree 4 , its derivative is calculated as follow:

$$
\begin{aligned}
\dot{V}(\mathbf{x})= & \left(3 a_{2}-4 a_{1}\right) x_{1}^{6}+\left(6 a_{3}-3 a_{2}\right) x_{1}^{5} x_{2} \\
& +\left(9 a_{4}-2 a_{3}\right) x_{1}^{4} x_{2}^{2}+\left(12 a_{5}-3 a_{2}-a_{4}-8 a_{1}\right) x_{1}^{3} x_{2}^{3} \\
& +\left(-6 a_{2}-6 a_{3}\right) x_{1}^{2} x_{2}^{4}+\left(-4 a_{3}-9 a_{4}\right) x_{1} x_{2}^{5}-\left(2 a_{4}-12 a_{5}\right) x_{2}^{6}
\end{aligned}
$$

To solve the problem using the algebraic method, we select the vector $Z$ as follows:

$Z=\left[\begin{array}{lllllll}-1 & 0 & -1 & 0 & -1 & 0 & -1\end{array}\right]^{T}$

Then, by using the Eq. (24), we obtain the following coefficients:

$a_{1}=0.184, \quad a_{2}=0.054, \quad a_{3}=0.090, \quad a_{4}=-0.069, \quad a_{5}=0.113$ 


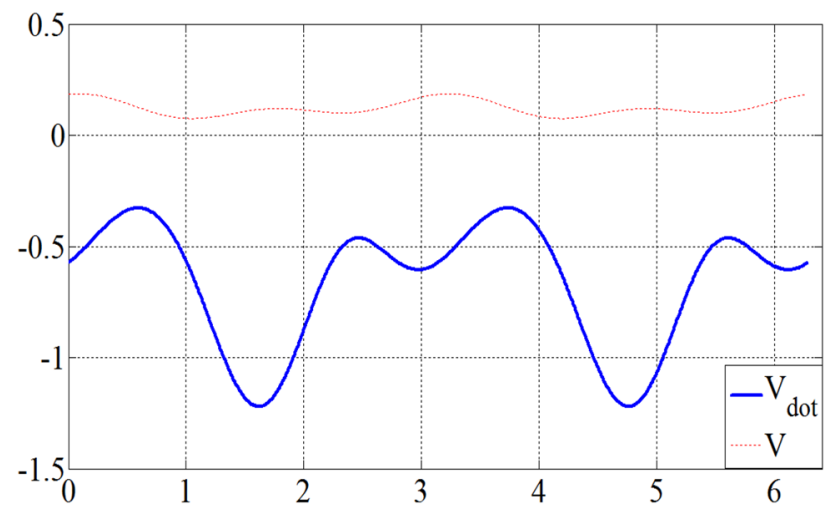

Fig. 2 The value of $V(X)$ and $\dot{V}(X)$ on the interval $\theta \in(0,2 \pi)$

So the $\dot{V}(X)$ form will be obtained as follows:

$$
\begin{aligned}
\dot{V}(\mathbf{x})= & -0.5722 x_{1}^{6}+0.3753 x_{1}^{5} x_{2}-0.8052 x_{1}^{4} x_{2}^{2} \\
& -0.2138 x_{1}^{3} x_{2}^{3}-0.867 x_{1}^{2} x_{2}^{4}+0.266 x_{1} x_{2}^{5}-1.2138 x_{2}^{6}
\end{aligned}
$$

In Fig. 2, the value of $\dot{V}(\mathbf{x})$ for, $\mathbf{x}=[\sin \theta, \cos \theta]$ is plotted on the unit circle. As is evident from the Fig. $2, \dot{V}(\mathbf{x})$ is negative definite. Also, the $V(\mathbf{x})$ function is obtained as follows:

$V=0.184 x_{1}^{4}+0.0546 x_{1}^{3} x_{2}+0.0899 x_{1}^{2} x_{2}^{2}-0.0695 x_{1} x_{2}^{3}+0.1127 x_{2}^{4}$

As shown in Fig. $2, V(X)$ is a certain positive function, and therefore, by referring to the theory 1 , the asymptotic stability of equilibrium point of the system Eq. (10) is proved.

Example 2 Consider the homogeneous system described in Eq. (15) with the following parameters values:

$b_{11}=2, \quad b_{12}=5, \quad b_{21}=-4, \quad b_{22}=1$

To analyze the stability of the equilibrium point of the system by using the algebraic method, by choosing the Lyapunov function presented in Eq. (16) and using Eq. (20) for the $H$ matrix, and taking into account the Eq. (29) for the $Z$ vector, the following value is obtained for the vector of unknown coefficients through the Eq. (24):

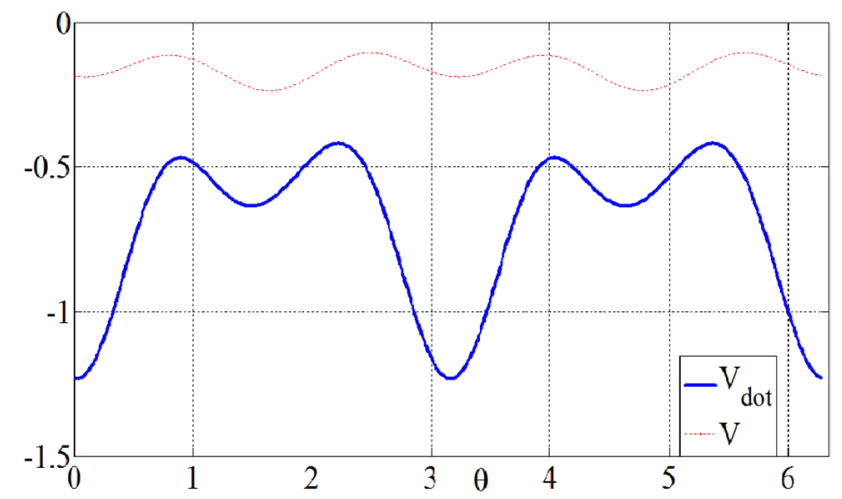

Fig. 3 The value of $V(X)$ and $\dot{V}(X)$ on the interval $\theta \in(0,2 \pi)$

Example 3 Consider the following homogeneous system of degree 2:

$\left\{\begin{array}{c}\dot{x}_{1}=x_{1}^{2}+x_{2}^{2} \\ \dot{x}_{2}=2 x_{1} x_{2}\end{array}\right.$

By selecting the Lyapunov candidate function as follows:

$V(x)=a_{1} x_{1}^{3}+a_{2} x_{1}^{2} x_{2}+a_{3} x_{1}^{1} x_{2}^{2}+a_{4} x_{2}^{3}$

And calculating its derivative along the system's answers, the following expressions are obtained for $V(\mathbf{x})$ and $\dot{V}(\mathbf{x})$ :

$\dot{V}(\mathbf{x})=-0.8148 x_{1}^{4}-1.185 x_{1}^{2} x_{2}^{2}-0.0741 x_{2}^{4}$

$V(\mathbf{x})=-0.2716 x_{1}^{3}-0.0741 x_{1} x_{2}^{2}$

where the algebraic method is used with the following $Z$ -vector.

$Z=\left[\begin{array}{lllll}-1 & 0 & -1 & 0 & -1\end{array}\right]^{T}$

By drawing the functions $V(\mathbf{x})$ and $\dot{V}(\mathbf{x})$ on a circle around the origin as it is clear in Fig. 4 , while $\dot{V}(\mathbf{x})$ is negative definite, $V(\mathbf{x})$ changes sign. By using Theorem 1, we find the instability of the equilibrium point of system Eq. (33).

$a_{1}=-0.054, \quad a_{2}=-0.063, \quad a_{3}=-0.032, \quad a_{4}=0.064, \quad a_{5}=-0.006$

By plotting $V(\mathbf{x})$ and $\dot{V}(\mathbf{x})$ functions on the unit circle around the origin on the phase space page, as shown in Fig. 3 , while $\dot{V}(\mathbf{x})$ is negative definite, $V(\mathbf{x})$ is also a negative definite function. By using the Theorem 1, we can conclude that the equilibrium point of the studied system is unstable.
Example 4 In this example, we reconsider the system presented in Eq. (10) and use the geometric method and in the case of the Theorem 1, we analyze the stability of its equilibrium point. We consider the initial Lyapunov function as the Eq. (16), whose derivative along the solution system, is as follows: 


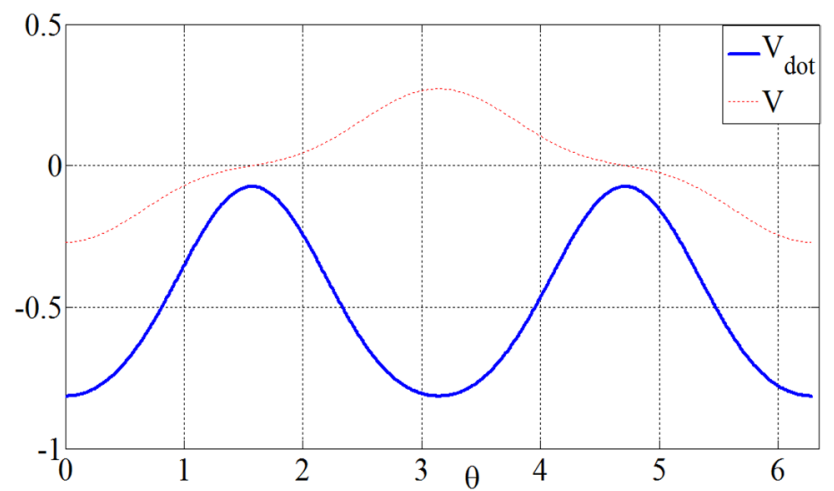

Fig. 4 The value of $v(x)$ and $\dot{V}(X)$ on the interval $\theta \in(0,2 \pi)$

$$
\begin{aligned}
\dot{V}(\mathbf{x})= & \left(-8 x_{1}^{3} x_{2}^{3}-4 x_{1}^{6}\right) a_{1}+\left(3 x_{1}^{6}-3 x_{1}^{5} x_{2}-3 x_{1}^{3} x_{2}^{3}-6 x_{1}^{2} x_{2}^{4}\right) a_{2} \\
& +\left(6 x_{1}^{5} x_{2}-2 x_{1}^{4} x_{2}^{2}-6 x_{1}^{2} x_{2}^{4}-4 x_{1} x_{2}^{5}\right) a_{3} \\
& +\left(9 x_{1}^{4} x_{2}^{2}-x_{1}^{3} x_{2}^{3}-9 x_{1} x_{2}^{5}-2 x_{2}^{6}\right) a_{4}+\left(12 x_{1}^{3} x_{2}^{3}+12 x_{2}^{6}\right) a_{5}
\end{aligned}
$$

To use the geometric method, we select 6 points on the unit circle in the form of $\left(a_{1} \ldots a_{5}\right)$, there will be an equation with five unknowns which, by means of Eq. (27), the amount of the coefficients are as follows:

$a_{1}=0.452, \quad a_{2}=0.269, \quad a_{3}=0.377$,

$a_{4}=-0.200, \quad a_{5}=0.109$

By placing these coefficients in $V(\mathbf{x})$ and $\dot{V}(\mathbf{x})$ and drawing the obtained functions in the distance $\theta \in(0,2 \pi)$, Fig. 5 will be obtained, where $N=6$.

As is shown in Fig. 5 , the value $\dot{V}(\mathbf{x})$ is always negative and $V(\mathbf{x})$ is always positive, according to the Theorem 1 , the

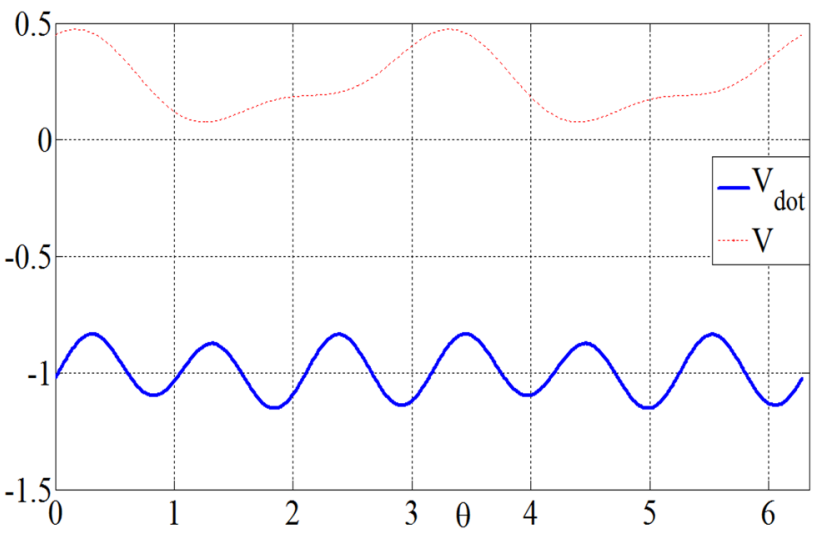

Fig. 6 The value of $V(X)$ and $\dot{V}(X)$ on the interval $\theta \in(0,2 \pi)$ for $N=25$

equilibrium point of the system is asymptotically stable. As indicated, Fig. 5 is plotted for $N=6$. Now by increasing the $N$ by 25 , Fig. 6 shows that a significant change in the values of $V(\mathbf{x})$ will not be achieved, while the shape $\dot{V}(\mathbf{x})$ around -1 is getting smoother that this is also due to the increase in the number of selected points in calculating the coefficient vector in the geometric method.

So far, assuming $p=4$ has been worked out. Now, we want to see the effect of increasing $p$ on the $\dot{V}(\mathbf{x})$ oscillation range around -1 . So we repeat the problem again for $p=8$, which leads to the definition of the following function:

$$
\begin{aligned}
V(\mathbf{x})= & a_{1} x_{1}^{8}+a_{2} x_{1}^{7} x_{2}+a_{3} x_{1}^{6} x_{2}^{2}+a_{4} x_{1}^{5} x_{2}^{3}+a_{5} x_{1}^{4} x_{2}^{4} \\
& +a_{6} x_{1}^{3} x_{2}^{5}+a x_{1}^{2} x_{2}^{6}+a_{8} x_{1} x_{2}^{7}+a_{9} x_{2}^{8}
\end{aligned}
$$

By using the geometric method we will have:

$$
\begin{aligned}
\dot{V}(x)= & -1.015 x_{1}^{10}+0.03 x_{1}^{9} 3 x_{2}-4.249 x_{1}^{8} x_{2}^{2}-0.41 x_{1}^{7} x_{2}^{3}-13.49 x_{1}^{6} x_{2}^{4} \\
& +1.044 x_{1}^{5} x_{2}^{5}-6.538 x_{1}^{4} x_{2}^{6}-0.598 x_{1}^{3} x_{2}^{7}-5.724 x_{1}^{2} x_{2}^{8}+0.059 x_{1} x_{2}^{9}-0.984 x_{2}^{10}
\end{aligned}
$$

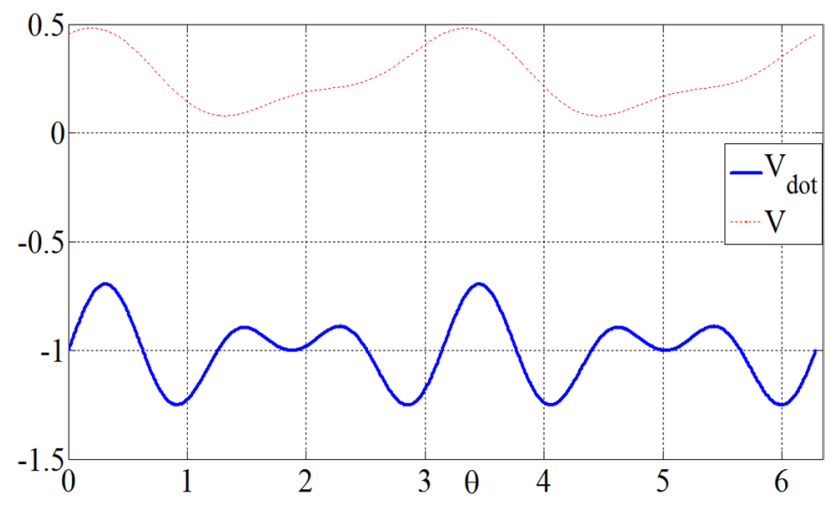

Fig. 5 The value of $V(X)$ and $\dot{V}(X)$ on the interval $\theta \in(0,2 \pi)$ for $N=6$

\section{SN Applied Sciences}

As shown in Fig. 7, the oscillation in $\dot{V}(\mathbf{x})$ is reduced, which means $\dot{V}(\mathbf{x})$ is nearer to the optimal value -1 .

Thus, we can say by increasing the degree of the initial Lyapunov function, can more accurately obtain a certain negative $\dot{V}(X)$. 


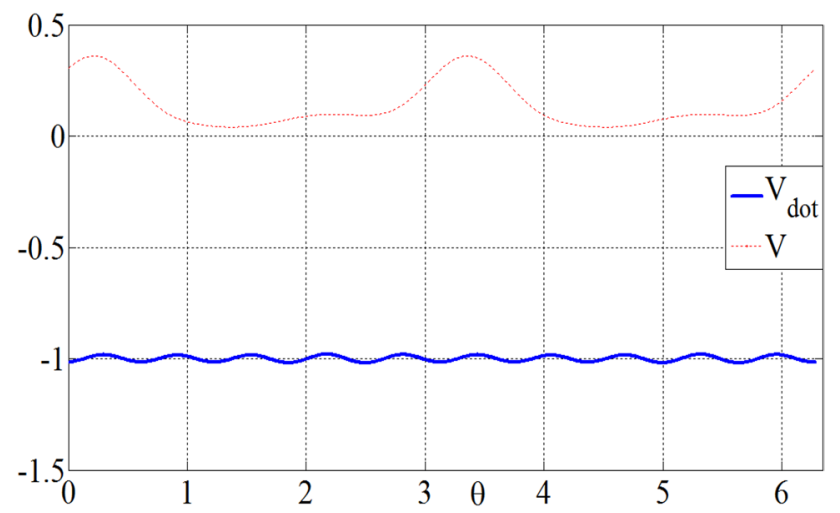

Fig. 7 The value of $V(X)$ and $\dot{V}(X)$ on the interval $\theta \in(0,2 \pi)$ for $N=25, p=8$

\section{Conclusion and further directions}

The Lyapunov stability and instability theorems present a standard method for analyzing the stability of nonlinear systems. This article by considering the common part of the previous theorems, which is being negative definite of the derivative of the Lyapunov candidate function, presented these cases in the form of a theorem, which is based on determining the sign of the initial function. Therefore, the approach of this paper emphasizes on the being negative definite of its derivative in the analysis of the stability of nonlinear systems, rather than focusing on the being positive definite on the significance of the Lyapunov candidate function. This method forms the Lyapunov candidate function as a linear combination of some base functions a $p$-form with unknown coefficients. The coefficients of this linear composition must be found in such a way as derivative of the candidate function Lyapunov be a negative definite function. For this purpose, two methods, called algebraic and geometric methods, were presented to calculate the unknown coefficients in homogeneous systems, based on the least squares method.

Although we have shown progress to find unknown coefficients for the Lyapunov function in the homogenous system, we can expand it to other nonlinear systems, and it could be an open question. Further work should include the development of stability analysis for a new nonlinear system. Besides, we can implement this solution in any branch of science.

\section{Compliance with ethical standards}

Conflict of interests The author declare that they have no competing interests.

\section{References}

1. Khalil H (2002) Nonlinear systems. Prentice Hall, Upper Saddle River

2. Kim H, Guo P, Zhu M, Liu P (2017)Attack-resilient estimation of switched nonlinear cyber-physical systems. In: 2017 American control conference (ACC), Seattle, pp 4328-4333

3. Fawzi H, Tabuada P, Diggavi S (2014) Secure estimation and control for cyber-physical systems under adversarial attacks. IEEE Trans Autom Control 59(6):1454-1467

4. Kim H, Guo P, Zhu M, Liu P (2016) Attack-resilient estimation of switched nonlinear cyber-physical systems. arXiv preprint http://arxiv.org/abs/1609.03600

5. Pasqualetti F, Dörfler F, Bullo F (2013) Attack detection and identification in cyber-physical systems. IEEE Trans Autom Control 58(11):2715-2729

6. Sande José, Peña Enrique, Maciñeira Enrique (2018) Stability of breakwater roundhead protected with a Cubipod single-layer armor. Appl Ocean Res 79:36-48

7. Keshtegar Behrooz (2016) Chaotic conjugate stability transformation method for structural reliability analysis. Comput Methods Appl Mech Eng 310:866-885

8. Chen G, Guo Z (2019) Observer-based distributed control and synchronization analysis of inverter-based nonlinear power systems. Nonlinear Dyn 1-23

9. Mobayen Saleh, Baleanu Dumitru (2016) Stability analysis and controller design for the performance improvement of disturbed nonlinear systems using adaptive global sliding mode control approach. Nonlinear Dyn 83(3):1557-1565

10. Hafstein SF (2007) An algorithm for constructing Lyapunov functions. Electron J Differ Equ Monograph 08

11. Ji Z, Wu W, Feng Y, Zhang G (2013) Constructing the Lyapunov function through solving positive dimensional polynomial system. J Appl Math 13, 859578

12. Schwartz CA, Yan A (1997) Construction of Lyapunov functions for nonlinear systems using normal forms. J Math Anal Appl 216(2):521-535

13. Papachristodoulou A, Prajna S (2002) On the construction of Lyapunov functions using the sum of squares decomposition. In: Proceedings of IEEE CDC

14. Fisher J, Bhattacharya R (2009) Analysis of partial stability problems using sum of squares techniques. Automatica 45(3):724-730

15. Matsue K, Hiwaki T, Yamamoto N (2017) “On the construction of Lyapunov functions with computer assistance. J Comput Appl Math 319:385-412

16. Hancock EJ, Papachristodoulou A (2013) Generalised absolute stability and sum of squares. Automatica 49(4):960-967

17. Wang Z, Liu X, Liu K, Li S, Wang H (2017) Backstepping-based Lyapunov function construction using approximate dynamic programming and sum of square techniques. IEEE Trans Cybern 47(10):3393-3403

18. Ling Q, Jin XL, Wang Y, Li HF, Huang ZL (2013) Lyapunov function construction for nonlinear stochastic dynamical systems. Nonlinear Dyn 72(4):853-864

19. Anghel M, Milano F, Papachristodoulou A (2013) Algorithmic construction of Lyapunov functions for power system stability analysis. IEEE Trans Circuits Syst I Regul Pap 60(9):2533-2546

20. Hafstein S, Kellett CM, Li H (2014) Continuous and piecewise affine Lyapunov functions using the Yoshizawa construction. In: American control conference (ACC)

21. Meigoli V, Ansari M, Barhaghtalab MH (2017) Stability analysis of non-linear dynamical homogeneous systems based on Lyapunov function constructed of linear combination of basic functions, Sindhological Studies, vol 2017, no 1 
22. Bouzaouache $H$, Braiek NB (2008) On the stability analysis of nonlinear systems using polynomial Lyapunov functions. Math Comput Simul 76(5-6):316-329

23. Shen Y, Xia X (2011) Global asymptotical stability and global finite-time stability for nonlinear homogeneous systems. IFAC Proc Vol 44(1):4644-4647

24. Haimo VT (1986) An algebraic approach to nonlinear stability. Nonlinear Anal. Theory Methods Appl. 10(7):683-692

25. Baillieul J (1980) The geometry of homogeneous-polynomial dynamical systems. Nonlinear Anal Theor Merhods Appl 4(5):879-900

26. Rosier $L$ (1992) Homogeneous Lyapunov function for homogeneous continuous vector field. Syst Control Lett 19:467-473
27. Kawski M (1990) Homogeneous feedback stabilization. In: Conte G, Perdon AM, Wyman B (eds) New Trends in Systems Theory. Birkhauser, Boston, pp 464-471

28. Slotine JJ, Li W (1991) Applied nonlinear control. Pearson, London

29. Mayer C (2000) Matrix analysis and applied linear algebra. SIAM, New Delhi

Publisher's Note Springer Nature remains neutral with regard to jurisdictional claims in published maps and institutional affiliations. 